\title{
Uncovering the transcriptomic and epigenomic landscape of nicotinic receptor genes in non-neuronal tissues
}

Bo Zhang ${ }^{1,2^{*}} \mathbb{D}$, Pamela Madden ${ }^{3}$, Junchen $\mathrm{Gu}^{2}$, Xiaoyun Xing ${ }^{2}$, Savita Sankar ${ }^{1}$, Jennifer Flynn ${ }^{2}$, Kristen Kroll and Ting Wang ${ }^{2^{*}}$

\begin{abstract}
Background: Nicotinic acetylcholine receptors (nAChRs) play an important role in cellular physiology and human nicotine dependence, and are closely associated with many human diseases including cancer. For example, previous studies suggest that nAChRs can re-wire gene regulatory networks in lung cancer cell lines. However, the tissue specificity of nAChRs genes and their regulation remain unexplored.

Result: In this study, we integrated data from multiple large genomic consortiums, including ENCODE, Roadmap Epigenomics, GTEx, and FANTOM, to define the transcriptomic and epigenomic landscape of all nicotinic receptor genes across many different human tissues and cell types. We found that many important nAChRs, including CHRNA3, CHRNA4, CHRNA5, and CHRNB4, exhibited strong non-neuronal tissue-specific expression patterns. CHRNA3, CHRNA5, and CHRNB4 were highly expressed in human colon and small intestine, and CHRNA4 was highly expressed in human liver. By comparing the epigenetic marks of CHRNA4 in human liver and hippocampus, we identified a novel liverspecific transcription start site (TSS) of CHRNA4. We further demonstrated that CHRNA4 was specifically transcribed in hepatocytes but not transcribed in hepatic sinusoids and stellate cells, and that transcription factors HNF4A and RXRA were likely upstream regulators of CHRNA4. Our findings suggest that CHRNA4 has distinct transcriptional regulatory mechanisms in human liver and brain, and that this tissue-specific expression pattern is evolutionarily conserved in mouse. Finally, we found that liver-specific CHRNA4 transcription was highly correlated with genes involved in the nicotine metabolism, including CYP2A6, UGT2B7, and FMO3. These genes were significantly downregulated in liver cancer patients, whereas CHRNA4 is also significantly down-regulated in cancer-matched normal livers.
\end{abstract}

Conclusions: Our results suggest important non-neuronally expressed nicotinic acetylcholine receptors in the human body. These non-neuronal expression patterns are highly tissue-specific, and are epigenetically conserved during evolution in the context of non-conserved DNA sequence.

Keywords: Nicotinic acetylcholine receptors, CHRNA4, Epigenetics, Tissue-specificity, Liver, Evolution

\footnotetext{
*Correspondence: bzhang29@wustl.edu; twang@genetics.wustl.edu

${ }^{1}$ Center of Regenerative Medicine, Department of Developmental Biology,

Washington University School of Medicine, Room 3212, 4515 McKinley

Research Building, 4515 McKinley Ave, St. Louis, MO 63110, USA

${ }^{2}$ Center for Genome Sciences and Systems Biology, Department of Genetics,

Washington University School of Medicine, Room 5211, 4515 McKinley

Research Building, 4515 McKinley Ave, St. Louis, MO 63110, USA

Full list of author information is available at the end of the article
}

(c) The Author(s). 2017 Open Access This article is distributed under the terms of the Creative Commons Attribution 4.0 International License (http://creativecommons.org/licenses/by/4.0/, which permits unrestricted use, distribution, and reproduction in any medium, provided you give appropriate credit to the original author(s) and the source, provide a link to the Creative Commons license, and indicate if changes were made. The Creative Commons Public Domain Dedication waiver (http://creativecommons.org/publicdomain/zero/1.0/) applies to the data made available in this article, unless otherwise stated. 


\section{Background}

Tobacco dependence (mainly through cigarette smoking) is a major global health problem and is a main cause of cancer and cancer-related death throughout the world. Nicotine, the biologically active substance in tobacco, promotes the addiction of smoking behaviors through activation of nicotinic acetylcholine receptors (nAChRs) [1]. These nAChRs typically combine to form fast, ionotropic cationic nicotinic receptor channels. Pentameric nAChRs usually consist of five subunits, with an overall molecular weight of $290 \mathrm{kDa}$. nAChRs subunits are broadly classified into two subtypes: muscle-type nicotinic receptors, including $\alpha 1, \beta 1, \gamma, \delta$, and $\varepsilon$ subunits, and neuronal-type nicotinic receptors, including $\alpha 2-\alpha 10$ and $\beta 2-\beta 4$ subunits. Neuronal-type nicotinic receptors are usually found in the brain, and exhibit some similarities with GABAa receptors and glycine receptors [1]. In human brain, the $\alpha 4$ and $\beta 2$ subunits are predominantly expressed and form pentameric $(\alpha 4)_{3}(\beta 2)_{2}$ and $(\alpha 4)_{2}(\beta 2)_{3}$ nAChRs. Other nAChR subunits, including $\alpha 3, \alpha 5 \alpha 7, \beta 3$, and $\beta 4$ are also expressed in human brain, usually forming homomeric and heteromeric receptors [2].

Neuronal-type nAChRs are generally believed to function in the brain and contribute to nicotine dependence through reward pathways [3]. Interestingly, previous studies reported that several neuronal-type nAChRs are also expressed in lung cancer cells and intestinal epithelium cells [4-7]. However, the overall expression pattern of nAChRs in different human tissues is largely unknown. To gain the knowledge of tissue - specific regulation of nAChRs, we took advantage of resources generated by several large genomic consortiums that aim to functionally annotate the human genome, including the ENCODE project [8], Roadmap Human Epigenomics project [9], FANTOM project [10], and GTEx project [11]. By comparing and combining extensive genomic datasets produced by these consortiums, we were able to define a comprehensive transcriptomic and epigenomic landscape of $\mathrm{nAChR}$ genes and investigate the regulatory mechanisms governing activities of these important genes.

Surprisingly, our investigation revealed that many neuronal-type nicotinic receptor subunits were highly expressed in non-neuronal tissues. In particular, we identified liver-specific expression of CHRNA4, and colon- and intestine-specific expression of CHRNA3, CHRNA5, and CHRNB4. These tissue-specific expression patterns of nAChRs were consistent with tissuespecific epigenetic patterns of these genes. Additionally, we discovered a novel alternative promoter of CHRNA4 in human liver, through which transcription factors HNF4A and RXRA could directly regulate CHRNA4 expression in hepatocytes. Despite the lack of DNA sequence conservation at the liver-specific promoter of
CHRNA4 between rodents and hominoids, the liverspecific expression and regulatory mechanism of CHRNA4 seem to be evolutionarily conserved between human and mouse liver. These results suggest a genetically dynamic but epigenetically conserved evolutionary history of CHRNA4.

\section{Results}

Tissue-specific expression pattern of human nAChRs

To understand the expression pattern of $\mathrm{nAChR}$ subunits in human, we examined the mRNA expression levels of 13 nAChRs genes that encode 9 alpha-subunits and 4 beta-subunits. By analyzing mRNA-sequencing data from 27 different human tissues and cell types generated by the Roadmap Epigenomes project [9], we found that nAChRs varied widely in their expression in a tissue-dependent manner. For example, CHRNB1 was found to be highly expressed across multiple tissues (Fig. 1a). Surprisingly, while $C H R N B 2$, the beta-subunit of $\alpha 4 \beta 2$-containing nicotinic receptors, was found to only be highly expressed in brain tissues, CHRNA4, the most abundant nAChR alphasubunit in human brain [2], was highly expressed in human adult liver in addition to being highly expressed in brain (Fig. 1). This tissue-specific expression pattern was validated in an independent cohort based on the Genotype-Tissue Expression project (GTEx) [11] (Fig. 1b).

The CHRNA3-CHRNA5-CHRNB4 loci (chr15-q25.1) is the hotspot for genetic variants that are associated with heavy smoking and nicotine dependence [12-14]. While these genes exhibit expected expression in the brain, we found much higher expression levels of CHRNA3, CHRNA5, and CHRNB4 in colon and small intestine than in brain tissues. This expression pattern was also recapitulated by the GTEx datasets (Fig. 1b).

\section{Epigenetic profile predicts novel liver-specific alternative promoter for CHRNA4}

Tissue-specific epigenetic profiles of a gene are strong predictors of tissue-specific gene activity. Active histone modifications (for example, H3K4me1, H3K4me3, and $\mathrm{H} 3 \mathrm{~K} 27 \mathrm{ac}$ ) and DNA hypomethylation in promoter regions are hallmarks of active genes $[8,9,15]$. To understand the high expression of neuronal-type nAChRs in human non-neuronal tissues, we examined the epigenetic landscape around CHRNA3, CHRNA4, CHRNA5, CHRNB2, and CHRNB4 in human liver, hippocampus, CD34 hematopoietic stem cells, colon, and lung tissues using the WashU Epigenome browser [16, 17]. We found that tissue-specific expression of nAChRs was strongly associated with the tissue-specific active epigenetic marks around the gene promoter (Additional file 1: Figure S1). In human hippocampus, we detected strong H3K4me3 and H3K27ac signals around known transcription start sites (TSS) of CHRNA3, CHRNA5, 

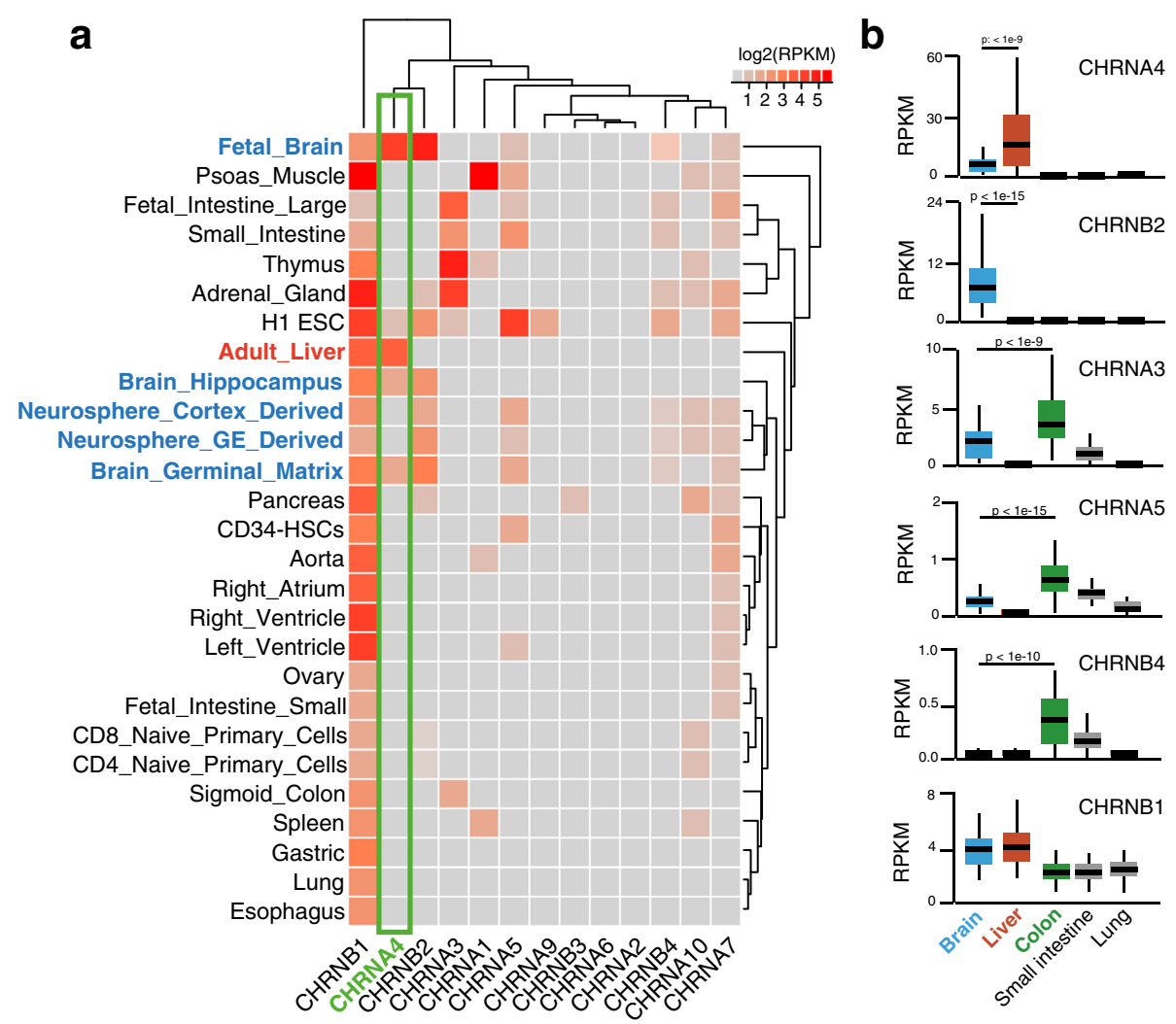

Fig. 1 Tissue-specific expression patterns of nicotinic acetylcholine receptors (nAChRs). a Heat map view of nAChRs expression patterns in 27 human tissue/cell types. Color scale indicates the expression level (RPKM) measured by RNA-seq from the Human Roadmap Epigenome project. $\mathbf{b}$ Expression level of CHRNA4, CHRNB2, CHRNA3, CHRNA5, CHRNAB4, and CHRNB1 in human brain, liver, colon, small intestine, and lung tissue. Y-axis indicates expression level (RPKM) measured by RNA-seq data from the GTEx project. Student $t$-test was performed to detect statistical significance

CHRNB2, CHRNB4, and CHRNA4 (Additional file 2: Figure. S2). Strikingly, in liver we detected very strong H3K4me3 and H3K27ac signals at $3.9 \mathrm{~kb}$ upstream of the known RefSeq TSS (Fig. 2a). This stunning promoter signature predicted a liver-specific, alternative promoter and/or transcription start site for CHRNA4.

Our prediction was confirmed using the Cap Analysis Gene Expression sequencing (CAGE-seq) data from the FANTOM5 project [10]. CAGE-seq identifies gene transcription start sites by sequencing the $5^{\prime}$ capped ends of mRNAs [18]. We found that the CAGE signal from human liver was only presented at $-3.9 \mathrm{~kb}$ upstream (chr20: 61,996,626-61,996,696) of the canonical TSS. In contrast, CAGE signal from brain was located around the known canonical CHRNA4 RefSeq TSS (chr20: 61,992,747-61,992,748) (Fig. 2a). As a control, we examined the histone modifications and CAGE signal for the CHRNA4 gene in human $\mathrm{CD} 34^{+}$hematopoietic stem cells (CD34-HSCs), where the gene is known to be silent (Fig. 1a). We did not observe enrichment of active histone modification marker (H3K27ac) at either brainspecific or liver-specific promoter regions of CHRNA4 in HSCs, nor did we observe CAGE signal in these regions
(Fig. 2a). We also noticed that the single nucleotide polymorphism (SNPs) around CHRNA4 were not associated with CHRNA4 expression level in brain hippocampus, but 4 SNPs were strongly associated with CHRNA4 expression level in human liver (Fig. 2a, processed expression quantitative trait loci (eQTL) data was downloaded from GTEx Project).

We also examined the DNA methylation level of both brain-specific and liver-specific CHRNA4 promoter regions $(+/-500 \mathrm{bp}$ of the TSS). The liver-specific CHRNA4 promoter was significantly hypomethylated in human liver and hypermethylated in both brain and CD34-HSCs (Fig. 2b), while the brain-specific promoter was hypermethylated in liver and hypomethylated in the hippocampus and CD34-HSCs (Fig. 2b). Additionally, we validated the expression level of CHRNA4 using $\mathrm{q}$ RT-PCR, and confirmed that the expression of CHRNA4 is about five-fold higher in human liver than it is in human brain, and CHRNA4 is not expressed in B cell lymphocyte (Fig. 2c). Taken together, our results reveal a distinctive promoter usage of the CHRNA4 gene in human liver and brain, highlighting a novel tissue-specific regulatory mechanism. 


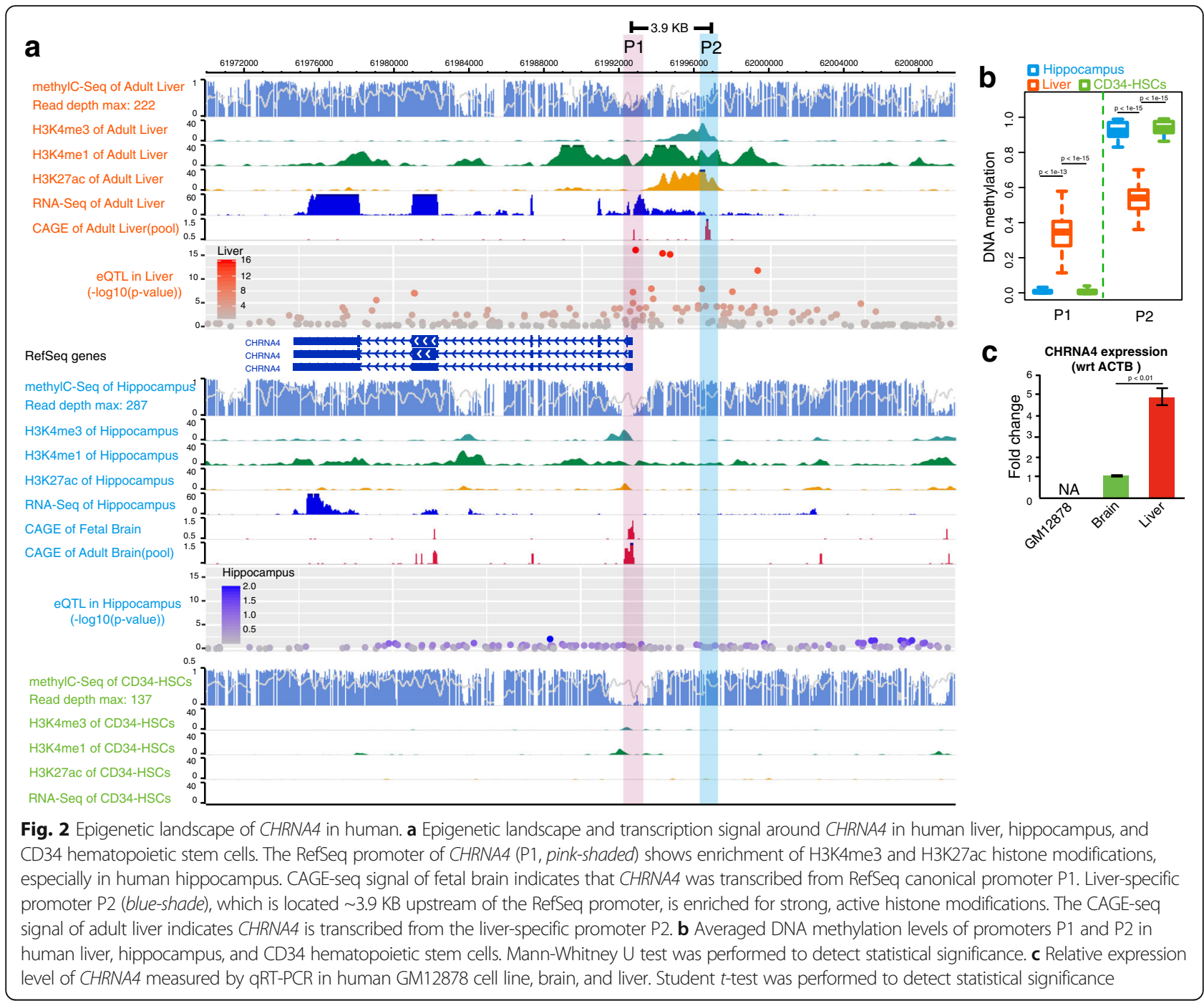

\section{Conserved expression and epigenetic patterns of Chrna4 in mouse}

Nicotinic acetylcholine receptors play important roles in the central nervous system, and are highly conserved from Drosophila to vertebrates [19]. We next determined if the unexpected liver-specific expression of CHRNA4 observed in human was an evolutionarily conserved phenomenon. To this end we took advantage of the data resources produced by the mouseENCODE consortium and FANTOM5 $[10,20]$ by integrating gene expression data, epigenomic data, and RNA polymerase II (Pol-2) ChIP-seq data, with CAGE-seq data from mouse brain and liver. We found that the epigenetic landscape between human and mouse is highly conserved in a tissue-specific manner surrounding the CHRNA4 /chrna4 gene in human and mouse, respectively. In mouse liver, active epigenetic modifications, including the H3K27ac signal, PoI-2 ChIP-seq signals, and DNaseI hypersensitivity signal, were highly enriched in a region $\sim 4.8 \mathrm{~kb}$ upstream of the RefSeq annotated Chrna4 TSS. In contrast, in mouse brain, the active epigenetic modifications were depleted in this region, but enriched around the canonical promoter (Additional file 2: Figure S2A). CAGE-seq data also support the alterative TSS usages between brain and liver (Additional file 2: Figure S2A, Fig. 4b). Specifically, the CAGE-seq signals were not found in hepatic sinusoids and stellate cells but was only presented in hepatocytes (Fig. 4b). We further confirmed the higher expression of Chrna4 in mouse liver than in brain with RT-PCR (Additional file 2: Fig. S2B). These data strongly suggest that there exists a novel but evolutional conserved mechanism to regulate tissue-specific activities of CHRNA4/Chrna4 in human and mouse, and that this neuronal-type nAChR might play a conserved and uncharacterized role in liver.

Further, we checked the DNA sequence conservation of the liver-specific CHRNA4 and Chrna4 promoters. We found that the orthologous region of the human 
liver-specific CHRNA4 TSS is conserved only in hominoid monkeys, and is absent in many other monkeys (Rhesus, Baboon, macaque, and marmoset) and rodents (Fig. 3a). Conversely, the orthologous region of the mouse liver-specific Chrna4 TSS is highly conserved among rodents, primates and other mammals (Fig. $3 \mathrm{~b}$ ). In the human genome, we found a highly enriched H3K4me1 signal in the orthologous region of the mouse liver-specific Chrna4 TSS, which is located $\sim 2 \mathrm{~kb}$ upstream the human liver-specific CHRNA4 TSS (Fig. 2a, Fig. 4b). Such evidence suggests that a 'turn-over' event may have occurred during primate evolution, and may also suggest that the 'evolutionarily conserved' liverspecific expression of CHRNA4/Chrna4 evolved independently in hominoids and rodent animals.

\section{HNF4A and RXRA may be involved in liver-specific CHRNA4 expression}

To understand the liver-specific regulatory mechanism of CHRNA4, we examined the transcription factor binding events around the CHRNA4 promoter region. Over 20 different transcription factors were found to bind to the $10 \mathrm{~kb}$ region surrounding the CHRNA4 promoter, as determined by the ENCODE consortium (Additional file 3: Figure S3). Considering the liverspecific expression pattern of CHRNA4, we reasoned that the upstream transcription factors of CHRNA4 should have a similar liver-specific expression pattern. After examining the expression patterns of 22 transcription factors that had binding sites near the CHRNA4 promoter across 31 major human tissues, we identified HNF4A and RXRA as highly expressed in human livers (Fig. 4a) and with binding sites in the vicinity of the CHRNA4 promoter. We also analyzed ChIP-seq data for Hnf4a and Rxra in mouse, and found that Hnf4a and Rxra directly bind to the promoter region of Chrna4. Furthermore, an Rxra ChIP-seq peak directly overlapped with the mouse liver-specific TSS (Fig. 4b). These data indicate that HNF4A /Hnf4a and RXRA/Rxra could be important TFs regulating the liver-specific expression of CHRNA4 / Chrna4 in both human and mouse, providing a potential mechanistic explanation behind the observed 'conserved expression pattern' of CHRNA4/Chrna4 between rodents and primates. We identified 4 SNPs to be significantly associated with expression of CHRNA4 in human liver (eQTL), and all 4 SNPs were located within a liver specific regulatory element (Figs. $2 \mathrm{a}$ and $4 \mathrm{~b}$ ). Two of the SNPs, rs755203 and rs3810471, were directly under RXRA and HNF4A ChIP-seq peaks, although they did not overlap with predicted RXRA or HNF4A binding motifs. Three SNPs, rs6089899, rs755203, and rs3810471, were predicted to influence binding affinities of several transcription factors including KrüppelLike Factor (KLF) family members (Table 1).

\section{Liver-specific CHRNA4 expression is associated with nicotine metabolism pathway}

To understand the potential roles of CHRNA4 in the liver, we investigated the enriched functions of genes coexpressed with CHRNA4 in 119 normal human liver samples (GTEx V6). We found 705 genes were significantly and positively correlated with CHRNA4 expression, whereas another 380 genes were significantly and
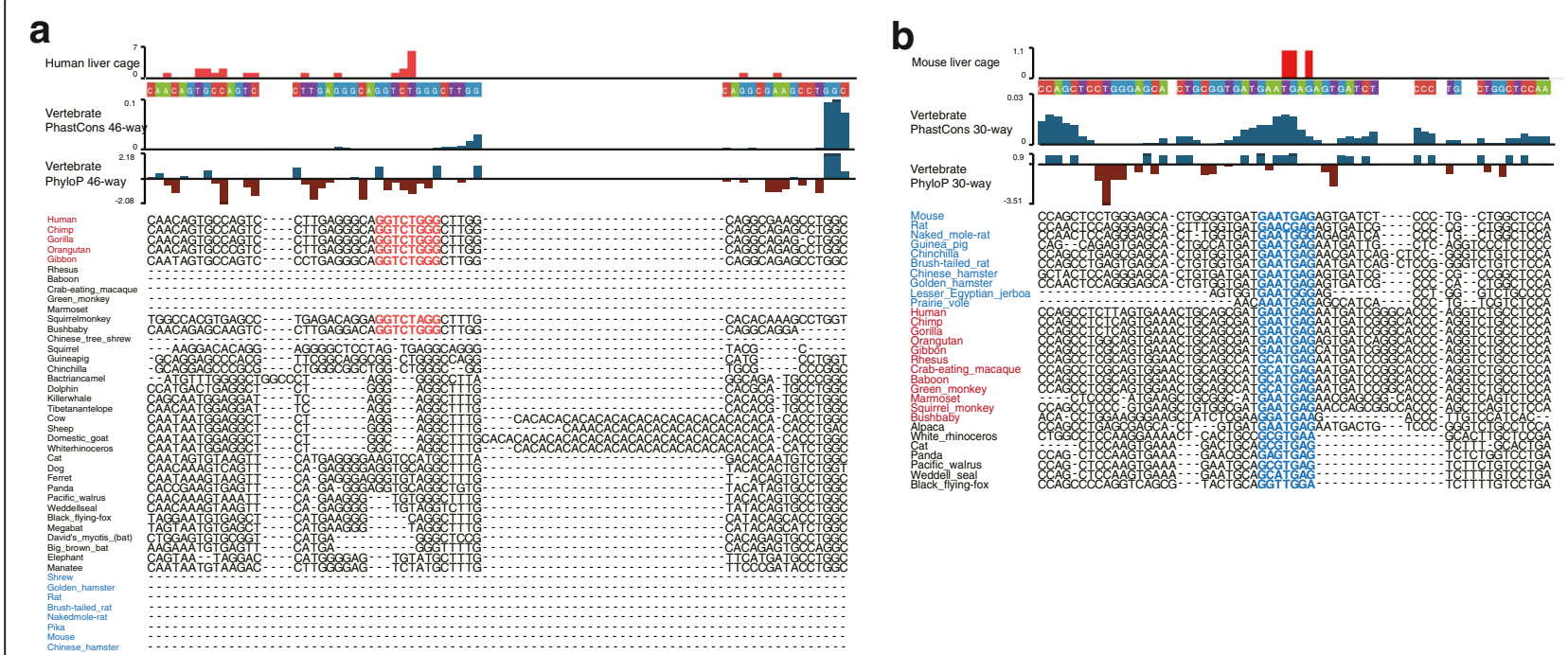

Fig. 3 Evolutionary dynamics of the CHRNA4 liver-specific promoter. a WashU EpiGenome Browser views of the conserved CHRNA4 liver-specific promoter in human and the orthologous sequence alignment in other vertebrate animals. The species without an orthologous sequence in this region were not displayed (except primates and rodents). $\mathbf{b}$ WashU EpiGenome Browser views of the conserved CHRNA4 liver-specific promoter in mouse and the orthologous sequence alignment in other vertebrate animals. The species without an orthologous sequence in this region were not displayed 


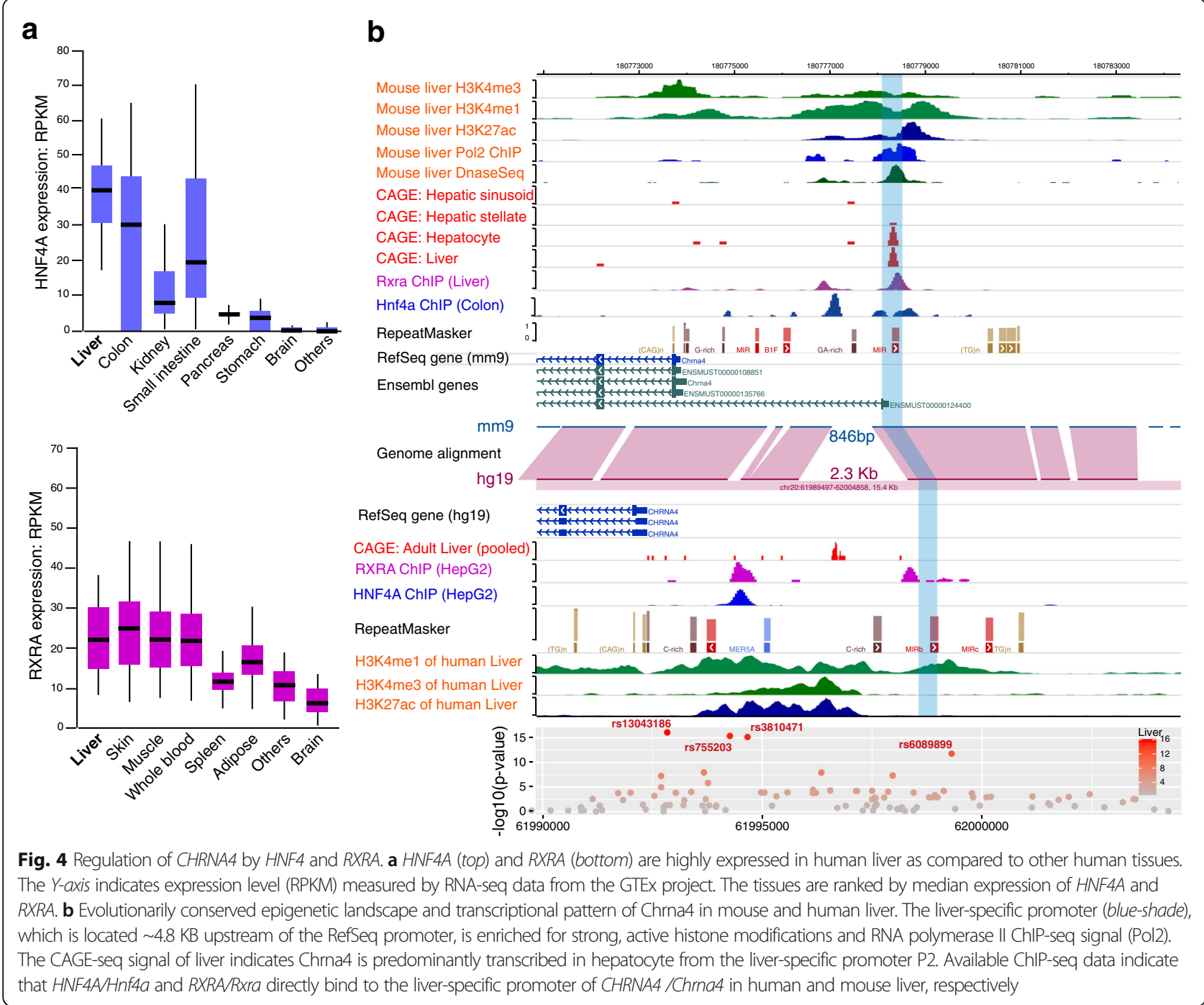

negatively correlated (Additional file 4: Figure S4, Additional file 5: Table S1). By using Ingenuity Pathway Analysis, we found that genes significantly positively correlated with CHRNA4 were highly enriched in several metabolic pathways, specifically in nicotine degradation (Fig. 5a, Additional file 6: Fig. S5). We further examined the expression level of important nicotine metabolism genes, and found expression of CYP2A6, UGT2B7, and FMO3 were significantly correlated with CHRNA4's expression in human liver (Fig. 5b). UGT1A6 exhibited anti-correlation but the correlation was less significant (Fig. 5b) and the expression level was relatively low (Additional file 7: Figure S6).

Smoking is generally believed to be a risk factor for liver cancer [21], we further examined the expression of CHRNA4 and nicotine metabolism - related genes in liver cancer samples by using TCGA liver hepatocellular carcinoma transcriptome data. With the exception of UGT1A6, all other genes were significantly less expressed in liver hepatocellular carcinoma as compared to normal liver samples (Fig. 5c). Furthermore, nicotine metabolism - related genes CYP2A6, FMO3, and UGT2B7 were expressed at a similar level in benign cancer-matched normal livers as in normal liver controls. However, CHRNA4 was significantly downregulated in cancer-matched normal livers (Fig. 5c).

\section{Discussion}

Nicotine is a lipophilic compound present at high levels in tobacco leaves, and can be easily absorbed in the bloodstream after smoking or chewing tobacco leaves. Nicotine can rapidly cross the blood-brain barrier and bind with high affinity to neuronal nicotinic acetylcholine receptors (nAChRs). nAChR activation excites target cells and mediates fast synaptic transmissions in autonomous ganglionic neurons in the brain [22, 23]. In human brain, CHRNA4 and CHRNB2, in the form of $(\alpha 4)_{3}(\beta 2)_{2}$, are the most abundant subunits of pentameric neuronal 
Table 1 Transcription factor motif analysis of SNPs associated with CHRNA4 liver-specific expression.

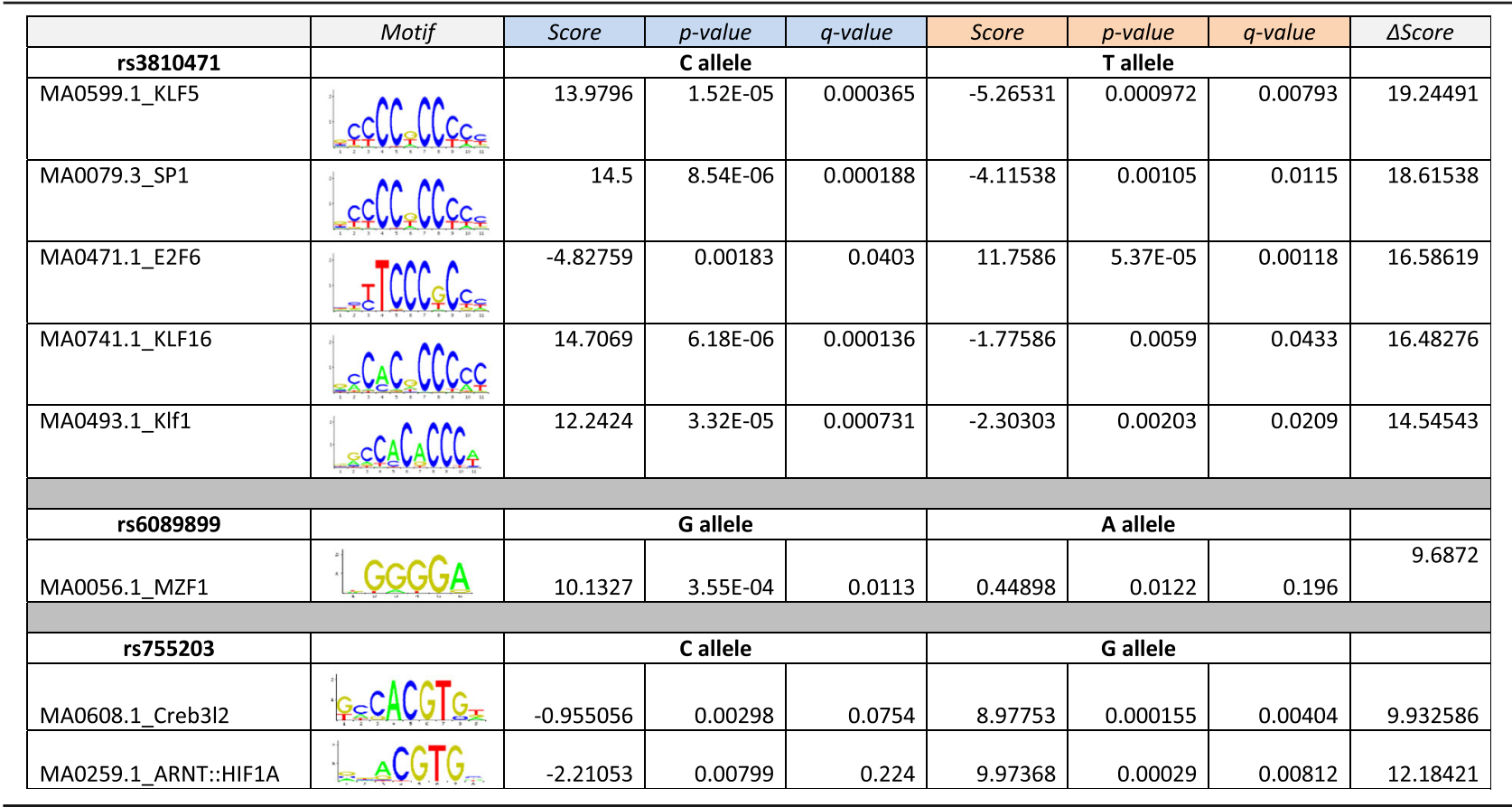

nicotinic receptors; however, other $\mathrm{nAChR}$ subunits ( $\alpha 3$, $\alpha 5, \alpha 7, \beta 2, \beta 3, \beta 4)$ also function as important components of homomeric/heteromeric receptor complexes [24]. All of these genes are associated with human smoking behaviors and nicotine addiction [6, 7, 25-33].

In an effort to define the tissue-specific epigenomic and transcriptomic landscape of nAChR genes, we discovered that CHRNA4 was highly expressed in human liver. Additionally, CHRNA3, CHRNA5, and CHRNB4 were highly expressed in colon and kidney. Expression of these neuronal-type nAChRs in non-neuronal tissues was strongly correlated with their tissue-specific epigenomic patterns. Further investigation led us to the discovery of a novel alternative promoter that regulates CHRNA4 transcription specifically in liver. Importantly, this regulatory mechanism is evolutionarily conserved, as we confirmed an almost identical pattern in mouse. Our analysis further suggests that transcription factors HNF4A and RXRA may play a role as the upstream regulators of CHRNA4, potentially orchestrating the coregulation of CHRNA4 and CYP2A6, a key gene involved in nicotine metabolism [34]. Thus, our results establish correlated regulation as well as deregulation between CHRNA4, a gene that encodes a nicotine acetylcholine receptor, and genes involved in nicotine metabolism, in the context of normal liver and liver cancer, opening doors for questioning CHRNA4's role in nicotine metabolism regulation. Considering the role of CHRNA4 in mediating nicotine's effect as a receptor, it is tempting to hypothesize that it might play a novel role as sensor in recognizing nicotine during its metabolism in liver (Fig. 5d). Although some evidence has suggested that individuals with reduced metabolic function of CYP2A6 smoke fewer cigarettes and have a shorter smoking duration [35], the functionality of CHRNA4 in both normal liver and hepatocellular carcinoma still need to be further intensively investigated.

Smoking behavior is associated with liver cancer [21]. However, the molecular mechanism underlying liver cancer and the usage of tobacco, specifically nicotine metabolism, remains a mystery. We found that the expression level of CHRNA4 and nicotine metabolism genes, including CYP2A6, FMO3, UGT2B7 were dramatically down-regulated in human hepatocellular carcinoma, suggesting disrupted nicotine metabolism in hepatocellular carcinoma. Interestingly, CHRNA4 expression was low in matched normal liver cells from patients with cancer. Our analysis put $H N F 4 A$ upstream of liver-specific expression of both CHRNA4 and CYP2A6, providing a potential mechanistic link between nicotine receptor and nicotine metabolism. HNF4A could be a key factor connecting nicotine metabolism and liver cancer. Previous studies showed that HNF4A was dramatically down-regulated or impaired in hepatocellular carcinoma [36, 37], and that forced expression of HNF4A in hepatocellular carcinoma cells could promote the transition of tumors towards a less invasive phenotype [38, 39]. Collectively, these findings suggest a potential connection between nicotine metabolism and 


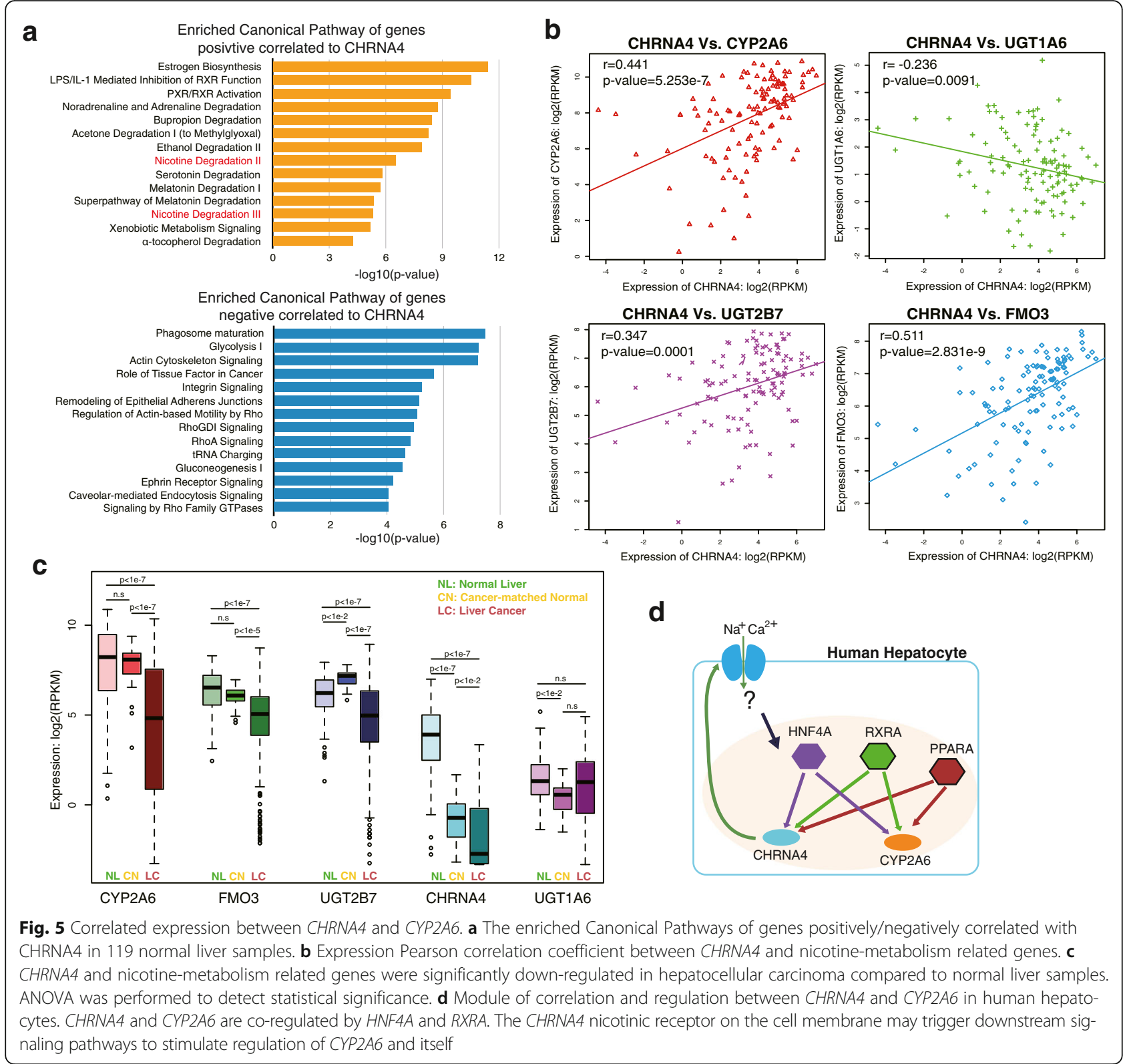

liver cancer. Understanding the molecular mechanism of such a connection could facilitate the study of smokingassociated hepatocellular carcinogenesis, and might shed new lights on clinical therapy of smoking cessations and liver cancer.

\section{Conclusion}

Nicotinic receptor genes are strongly associated with smoking behavior and nicotine dependence, and they are generally believed to be expressed specifically in the brain. In this work, by applying integrative genomics and comparative genomics, we described the expression and epigenetic landscape of nicotinic receptor genes in different non-neuronal human tissues. We found that nicotinic receptor alpha-4 (CHRNA4) was highly expressed in liver tissue, when comparing to brain and other tissues. We discovered a tissue-specific usage of an alternative CHRNA4 promoter in human brain and liver, identifying a novel liver-specific transcription start site of CHRNA4, located about $3.9 \mathrm{~KB}$ upstream of known canonical RefSeq TSS. This tissue-specific, alternative promoter usage pattern is conserved in mouse, suggesting a dynamic but epigenetically conserved evolutionary history of CHRNA4. We also found that the expression level of CHRNA4 was highly correlated with nicotine metabolism genes, and CHRNA4 was down-regulated in both hepatocellular carcinoma and tumor-adjacent normal liver tissues. Our study indicated that the integrative 
analysis of published data could reveal new directions in investigating the molecular mechanisms in nicotine sensing and metabolism in liver, and how disruption of these processes may play a role in hepatocellular carcinogenesis.

\section{Methods}

\section{Processing RNA-seq data of the Human Roadmap Epigenome Project}

Processed mRNA-seq datasets (aligned to human reference genome hg19) from 56 reference epigenomics were obtained from Roadmap epigenomics project through data portal (http://egg2.wustl.edu/roadmap/web_portal/). Expression of all nAChRs were isolated and visualized by using the gplots package in the $\mathrm{R}$ environment (Ver 3.2.2).

\section{Processing RNA-seq data from the TCGA project}

Processed mRNA-seq datasets (level 3, ht-seq reads count files) of 374 liver cancer samples and 50 cancermatched normal samples were downloaded from the Genomic Data Commons Data Portal (https://gdc-portal.nci.nih.gov/). The RPKM of each gene was calculated based on the annotated human gene length (GENCODE V23).

\section{Processing RNA-seq data from the GTEx project}

Processed mRNA-seq datasets (version V6, Reads Per Kilobase of transcript per Million mapped reads (RPKM) of genes) and data description files of 8555 samples were downloaded from the GTEx Portal (http://www.gtexportal.org/), including 119 liver samples, 320 lung samples, 149 colon sigmoid samples, 88 small intestine samples, and 1259 brain samples. Genes expression levels in different tissues (brain, liver, colon, small intestine, lung, and others) were plotted by using the ggplot2 package in the R environment.

\section{Co-expression correlation calculation}

One-hundred nineteen human liver transcriptomes in GTEx V6 data were used to calculate the co-expression correlation between CHRNA4 and other genes. Genes with an averaged expression level less than 1 RPKM were filtered out. The Pearson correlation coefficient between CHRNA4 and all other genes was calculated by using log-transformed RPKM with the cor function, and $p$-values were calculated using the cor.test function in the $\mathrm{R}$ environment. $p$-values were further corrected using the padjust function with the $\mathrm{BH}$ method in $\mathrm{R}$. Only the genes with an adjusted $p$-value less than 0.01 were considered as significantly correlated to CHRNA4, and were used to perform Ingenuity Pathway Analysis.

\section{Ingenuity pathway analysis (IPA)}

IPA (Ingenuity Systems, Redwood City, CA) software was used to determine the functional pathways and regulatory network models represented by the significantly correlated genes. The gene set was imported into IPA to perform a Core Analysis. The top 15 enriched canonical pathways were selected based on significance $(p$-value $<0.05)$.

\section{ChIP-seq data preprocessing and peak calling}

The raw reads of RXRA and HNF4A ChIP-seq data were downloaded from GEO, and aligned to the human genome (assembly hg19) and mouse genome (assembly mm9) using Bowtie V1.0.0 [40]. methylQA was used to process aligned bam files, isolate the non-redundant, uniquely aligned reads only, and extend the DNA fragments to $150 \mathrm{bp} \mathrm{[41].} \mathrm{Additional} \mathrm{file} \mathrm{8:} \mathrm{Table} \mathrm{S2} \mathrm{summa-}$ rizes the information for the individual ChIP-seq data sample files used in this study.

The histone ChIP-seq data for human tissues (liver, brain, lung, colon and CD34-HSC) were obtained from the Roadmap Epigenomics Project through a data portal (http://egg2.wustl.edu/roadmap/web_portal/). Raw-reads were aligned to human genome (assembly hg19) and mouse genome (assembly $\mathrm{mm} 9$ ) by using Bowtie V1.0.0 [40]. methylQA was used to process aligned bam files, isolate the non-redundant, uniquely aligned reads only, and extend the DNA fragments to $150 \mathrm{bp}$ [41].

The histone ChIP-seq data for mouse liver and cortex were obtained from the ENOCDE project through a data portal (https://www.encodeproject.org.). Raw-reads were aligned to the mouse genome (assembly $\mathrm{mm} 9$ ) using Bowtie V1.0.0 [40]. methylQA was used to process aligned bam files, isolate the non-redundant, uniquely aligned reads only, and extend the DNA fragments to $150 \mathrm{bp}$ [41].

The MACSv2.0.10 [42] peak caller was used to compare ChIP-seq signal to a corresponding ChIP-seq input control. To identify narrow regions of transcription factor/histone enrichment (peaks) across the genome, a q-value threshold of 0.01 was used. The bedGraph transcription factor ChIP-seq data files and histone ChIP-seq data files were visualized on the WashU Epigenome Browser.

\section{FANTOM5 CAGE data processing}

Cap Analysis Gene Expression by sequencing (CAGE-seq) data (bam files, liver and brain tissues for both human and mouse) generated by the FANTOM5 consortium were downloaded from FANTOM FTP (http://fantom.gsc.riken.jp/5/datafiles/latest). The bam files were then converted to a fastq file format, and aligned to human genome (assembly hg19) and mouse genome (assembly mm9) using Bowtie V1.0.0 [40]. The uniquely aligned reads were isolated using Samtools, and further transformed into bed files for visualization on the WashU Epigenome Browser.

\section{DNA methylation data processing}

Methylation calls for each CpG site were calculated using Whole-Genome Bisulfite Sequencing (WGBS) data 
for human tissues (liver, brain, lung, colon and CD34HSC) obtained from the Roadmap Epigenomics Project through a data portal (http://egg2.wustl.edu/roadmap/ web_portal/), and were visualized on the WashU Epigenome Browser. To measure the DNA methylation level of CHRNA4 promoters, methylation of CpG sites with a minimum of $10 \times$ coverage per site in a $1 \mathrm{~KB}$ region around the CHRNA4 TSS in human liver, brain, and CD34-HSC were isolated to generate boxplots and calculate statistical significance.

\section{eQTL data processing}

Tissue-specific eQTL data were downloaded from the GTEx Portal (http://www.gtexportal.org/). The SNPs located in CHRNA4 loci and associated with CHRNA4 (ENSG00000101204.11) in human liver and hippocampus were isolated using an in-house python script. The $p$-value of each SNP was negatively log-transformed and visualized on the WashU Epigenome Browser.

\section{Motif analysis}

Motif analyses were performed using the FIMO tool from the MEME suite [43]. The $10 \mathrm{bp}$ upstream and downstream each SNP were isolated using bedtools (getfasta) from the human reference genome (assembly hg19). Two allele-specific 21 bp DNA sequences were generated based on the allelic information obtained from dbSNP (build 144). Fimo was used to predict potential TF binding sites in two allele-specific 21 bp DNA sequences by using a PWM of 519 transcription factors downloaded from the JASPAR database [44].

\section{Genome alignment}

A genome alignment generated by blastz between human (hg19) and mouse (mm9) was obtained from the UCSC genome browser (http://hgdownload.cse.ucsc.edu/ downloads.html), and then visualized on the WashU Epigenome Browser to indicate the genome-level conservation at the CHRNA4/Chran4 loci. Multiple alignments of 45 vertebrate genomes of CHRNA4 promoters were directly generated by UCSC genome browser (http:// hgdownload.cse.ucsc.edu/).

\section{Quantitative real time PCR (qRT-PCR) analysis}

The qRT-PCR analyses were performed using the SuperScript VILO cDNA Synthesis Kit (Life Technologies) with iTaq Universal SYBR Green Supermix (Bio-Rad). All mouse and human brain and liver RNA was purchased from ZYAGEN. $500 \mathrm{ng}$ total RNA was used in a 20ul reverse transcription reaction. The cDNA obtained was diluted to a total volume of $100 \mathrm{ul}$ and stored at $-20{ }^{\circ} \mathrm{C}$. The primers for human CHRNA4 and mouse Chrna4 (listed in Additional file 8: Table S3) were synthesized by Integrated DNA Technologies. The qRT-
PCR was performed in a $20 \mathrm{ul}$ reaction mixture consisting of $2 \mathrm{ul}$ diluted cDNA, $0.2 \mathrm{uM}$ of each primer, and 10ul iTaq Universal SYBR Green Supermix. All amplifications were carried out in a Bio-Rad CFX96 Real-Time PCR Detection (Bio-Rad) with denaturation at $95^{\circ} \mathrm{C}$ for 30s, followed by 40 cycles at $95{ }^{\circ} \mathrm{C}$ for $5 \mathrm{~s}$ and $60{ }^{\circ} \mathrm{C}$ for 30s. A melting curve analysis was performed for each run to confirm the specificity of amplification and lack of primer dimers. The qRT-PCR experiments were always run in triplicate. The relative mRNA expression levels of target genes were quantified using the $2-\Delta \Delta C T$ methods as reported [45].

\section{Additional files}

Additional file 1: Figure S1. The epigenetic landscape around CHRNB4, CHRNA5, CHRNA3, CHRNB2, and CHRNA4 in human liver, CD34-HSC, brain, colon, and lung tissues. (PDF $288 \mathrm{~kb}$ )

Additional file 2: Figure S2. The epigenetic landscape and expression pattern of Chrna4 in mouse brain and liver. (PDF $115 \mathrm{~kb}$ )

Additional file 3: Figure S3. Transcription factors binding events around CHRNA4 promoter. (PDF $33.3 \mathrm{~kb}$ )

Additional file 4: Figure S4. Distribution of expression correlation coefficient between CYP2A6 and all genes in 119 human liver samples. (PDF $44.9 \mathrm{~kb}$ )

Additional file 5: Table S1. List of genes significantly correlated with CHRNA4 in human liver. (XLSX $123 \mathrm{~kb}$ )

Additional file 6: Figure S5. Enriched network modules in genes positively correlated to CHRNA4. (PDF $290 \mathrm{~kb}$ )

Additional file 7: Figure S6. The absolute expression level of CHRNA4, CYP2A6, UGT1A6, UGT2B7, and FMO3 in 119 human liver samples. (PDF $49.9 \mathrm{~kb}$ )

Additional file 8: Table S2-3. Dataset and primers used in this study. (DOCX $68.5 \mathrm{~kb})$

\section{Abbreviations}

CAGE-seq: Cap Analysis Gene Expression sequencing; CD34-HSCs: CD34+ Hematopoietic Stem Cells; eQTL: Expression Quantitative Trait Loci; GTEx: Genotype-Tissue Expression project; nAChRs: Nicotinic acetylcholine receptors; RPKM: Reads Per Kilobase of transcript per Million mapped reads; SNPs: Single Nucleotide Polymorphisms; TSS: Transcription Start Site

\section{Acknowledgement}

We acknowledge Dr. Andrew Heath for his helpful advice and discussions. We thank Caili Tong and Feiya Wang for generously helping with experiments. We acknowledge the Genotype-Tissue Expression (GTEx) project for public access of RNA-seq and eQTL data. The data used for the analyses described in this manuscript were obtained from the GTEx Portal on 10/10/ 16. This work was supported by the National Institution of Health [DA027995 to B.Z. and P.M., R01HG007354, R01HG007175, R01ES024992 to T.W.] and the American Cancer Society [RSG-14-049-01-DMC to B.Z. and T.W.].

\section{Availability of data and materials}

The RNA sequencing data and human epigenomics data were downloaded from the NIH RoadMap Epigenomics Data Portal (http://egg2.wustl.edu/ roadmap/web_portal/). Gene expression and eQTL data were downloaded from GTEx Data Portal (http://www.gtexportal.org/home/). The CAGE-seq data were downloaded from the FANTOM5 project (http://fantom.gsc.riken.jp/data/). The mouse epigenomics data and ChIP-seq data were down form Gene Expression Omnibus (http://www.ncbi.nlm.nih.gov/geo/query/acc.cgi?acc=GSE49847). 


\section{Authors' contributions}

Study was designed by BZ, PM and TW. Data was analyzed by BZ and JF. Experiment was performed by SS, JG, XX, and KK. Manuscript was written by $B Z, J F$, and TW. All authors have read and approved the manuscript.

\section{Competing interests}

The authors declare that they have no competing interests.

\section{Ethics approval and consent to participate}

Not Applicable.

\section{Publisher's Note}

Springer Nature remains neutral with regard to jurisdictional claims in published maps and institutional affiliations.

\section{Author details}

'Center of Regenerative Medicine, Department of Developmental Biology, Washington University School of Medicine, Room 3212, 4515 McKinley Research Building, 4515 McKinley Ave, St. Louis, MO 63110, USA. ${ }^{2}$ Center for Genome Sciences and Systems Biology, Department of Genetics, Washington University School of Medicine, Room 5211, 4515 McKinley Research Building, 4515 McKinley Ave, St. Louis, MO 63110, USA. ${ }^{3}$ Department of Psychiatry, Washington University School of Medicine, St. Louis, MO 63110, USA.

Received: 2 November 2016 Accepted: 23 May 2017

Published online: 05 June 2017

\section{References}

1. Cascio M. Structure and function of the glycine receptor and related nicotinicoid receptors. J Biol Chem. 2004;279:19383-6.

2. Albuquerque EX, Pereira EF, Alkondon M, Rogers SW. Mammalian nicotinic acetylcholine receptors: from structure to function. Physiol rev. 2009;89:73-120.

3. Laviolette SR, van der Kooy D. The neurobiology of nicotine addiction: bridging the gap from molecules to behaviour. Nat rev Neurosci. 2004;5:55-65.

4. Richardson CE, Morgan JM, Jasani B, Green JT, Rhodes J, Williams GT, et al. Megacystis-microcolon-intestinal hypoperistalsis syndrome and the absence of the alpha3 nicotinic acetylcholine receptor subunit. Gastroenterology. 2001;121:350-7

5. Zia S, Ndoye A, Nguyen VT, Grando SA. Nicotine enhances expression of the alpha 3, alpha 4, alpha 5, and alpha 7 nicotinic receptors modulating calcium metabolism and regulating adhesion and motility of respiratory epithelial cells. Res Commun Mol Pathol Pharmacol. 1997;97:243-62.

6. Plummer HK 3rd, Dhar M, Schuller HM. Expression of the alpha7 nicotinic acetylcholine receptor in human lung cells. Respir res. 2005;6:29.

7. Wang Y, Pereira EF, Maus AD, Ostlie NS, Navaneetham D, Lei S, et al. Human bronchial epithelial and endothelial cells express alpha7 nicotinic acetylcholine receptors. Mol Pharmacol. 2001;60:1201-9.

8. Consortium EP. An integrated encyclopedia of DNA elements in the human genome. Nature. 2012;489:57-74.

9. Roadmap Epigenomics C, Kundaje A, Meuleman W, Ernst J, Bilenky M, Yen $A$, et al. Integrative analysis of 111 reference human epigenomes. Nature. 2015;518:317-30.

10. Consortium F, the RP, Clst, Forrest AR, Kawaji H, Rehli M, Baillie JK, de Hoon MJ, et al. A promoter-level mammalian expression atlas. Nature. 2014;507:462-70.

11. Consortium GT. Human genomics. The genotype-tissue expression (GTEx) pilot analysis: multitissue gene regulation in humans. Science. 2015;348:648-60.

12. Berrettini W, Yuan X, Tozzi F, Song K, Francks C, Chilcoat H, et al. Alpha-5/ alpha-3 nicotinic receptor subunit alleles increase risk for heavy smoking. Mol Psychiatry. 2008;13:368-73.

13. Munafo MR, Timofeeva MN, Morris RW, Prieto-Merino D, Sattar N, Brennan P, et al. Association between genetic variants on chromosome $15 q 25$ locus and objective measures of tobacco exposure. J Natl Cancer Inst. 2012;104:740-8.

14. Saccone NL, Culverhouse RC, Schwantes-An TH, Cannon DS, Chen X, Cichon $S$, et al. Multiple independent loci at chromosome 15q25.1 affect smoking quantity: a meta-analysis and comparison with lung cancer and COPD. Plos Genet. 2010:6.

15. Zhang B, Zhou Y, Lin N, Lowdon RF, Hong C, Nagarajan RP, et al. Functional DNA methylation differences between tissues, cell types, and across individuals discovered using the M\&M algorithm. Genome res. 2013;23:1522-40.
16. Zhou X, Li D, Zhang B, Lowdon RF, Rockweiler NB, Sears RL, et al. Epigenomic annotation of genetic variants using the Roadmap Epigenome browser. Nat Biotechnol. 2015;33:345-6.

17. Zhou X, Maricque B, Xie M, Li D, Sundaram V, Martin EA, et al. The human Epigenome browser at Washington University. Nat Methods. 2011;8:989-90.

18. Kawaji H, Lizio M, Itoh M, Kanamori-Katayama M, Kaiho A, Nishiyori-Sueki H, et al. Comparison of CAGE and RNA-seq transcriptome profiling using clonally amplified and single-molecule next-generation sequencing. Genome res. 2014;24:708-17.

19. Bossy B, Ballivet M, Spierer P. Conservation of neural nicotinic acetylcholine receptors from drosophila to vertebrate central nervous systems. EMBO j. 1988;7:611-8.

20. Mouse EC, Stamatoyannopoulos JA, Snyder M, Hardison R, Ren B, Gingeras T, et al. An encyclopedia of mouse DNA elements (mouse ENCODE). Genome Biol. 2012:13:418.

21. Trichopoulos D, Bamia C, Lagiou P, Fedirko V, Trepo E, Jenab M, et al. Hepatocellular carcinoma risk factors and disease burden in a European cohort: a nested case-control study. J Natl Cancer Inst. 2011;103:1686-95.

22. Itoh M, Nakajima M, Higashi E, Yoshida R, Nagata K, Yamazoe Y, et al. Induction of human CYP2A6 is mediated by the pregnane $X$ receptor with peroxisome proliferator-activated receptor-gamma coactivator 1alpha. J Pharmacol exp Ther. 2006;319:693-702

23. Dani JA, Bertrand D. Nicotinic acetylcholine receptors and nicotinic cholinergic mechanisms of the central nervous system. Annu rev Pharmacol Toxicol. 2007:47:699-729.

24. Zoli M, Pistillo F, Gotti C. Diversity of native nicotinic receptor subtypes in mammalian brain. Neuropharmacology. 2015;96:302-11.

25. Feng $Y$, Niu T, Xing H, Xu X, Chen C, Peng S, et al. A common haplotype of the nicotine acetylcholine receptor alpha 4 subunit gene is associated with vulnerability to nicotine addiction in men. Am J hum Genet. 2004;75:112-21.

26. Li MD, Beuten J, Ma JZ, Payne TJ, Lou XY, Garcia V, et al. Ethnic- and genderspecific association of the nicotinic acetylcholine receptor alpha4 subunit gene (CHRNA4) with nicotine dependence. Hum Mol Genet. 2005;14:1211-9.

27. Coon H, Piasecki TM, Cook EH, Dunn D, Mermelstein RJ, Weiss RB, et al. Association of the CHRNA4 neuronal nicotinic receptor subunit gene with frequency of binge drinking in young adults. Alcohol Clin exp res. 2014:38:930-7.

28. Saccone NL, Wang JC, Breslau N, Johnson EO, Hatsukami D, Saccone SF, et al. The CHRNA5-CHRNA3-CHRNB4 nicotinic receptor subunit gene cluster affects risk for nicotine dependence in African-Americans and in EuropeanAmericans. Cancer res. 2009;69:6848-56.

29. Dawson A, Miles MF, Damaj MI. The beta2 nicotinic acetylcholine receptor subunit differentially influences ethanol behavioral effects in the mouse. Alcohol. 2013:47:85-94.

30. De Luca V, Wong AH, Muller DJ, Wong GW, Tyndale RF, Kennedy JL. Evidence of association between smoking and alpha7 nicotinic receptor subunit gene in schizophrenia patients. Neuropsychopharmacology. 2004;29:1522-6.

31. Sun X, Ritzenthaler JD, Zhong X, Zheng Y, Roman J, Han S. Nicotine stimulates PPARbeta/delta expression in human lung carcinoma cells through activation of PI3K/mTOR and suppression of AP-2alpha. Cancer res. 2009:69:6445-53.

32. Zeiger JS, Haberstick BC, Schlaepfer I, Collins AC, Corley RP, Crowley TJ, et al. The neuronal nicotinic receptor subunit genes (CHRNA6 and CHRNB3) are associated with subjective responses to tobacco. Hum Mol Genet. 2008;17:724-34.

33. Rice JP, Hartz SM, Agrawal A, Almasy L, Bennett S, Breslau N, et al. CHRNB3 is more strongly associated with Fagerstrom test for cigarette dependence-based nicotine dependence than cigarettes per day: phenotype definition changes genome-wide association studies results. Addiction. 2012;107:2019-28.

34. Pitarque $M$, Rodriguez-Antona $C$, Oscarson M, Ingelman-Sundberg $M$. Transcriptional regulation of the human CYP2A6 gene. J Pharmacol exp Ther. 2005:313:814-22.

35. Liu T, David SP, Tyndale RF, Wang H, Zhou Q, Ding P, et al. Associations of CYP2A6 genotype with smoking behaviors in southern China. Addiction. 2011;106:985-94.

36. Saha SK, Parachoniak CA, Ghanta KS, Fitamant J, Ross KN, Najem MS, et al. Mutant IDH inhibits HNF-4alpha to block hepatocyte differentiation and promote biliary cancer. Nature. 2014;513:110-4.

37. Bonzo JA, Ferry CH, Matsubara T, Kim JH, Gonzalez FJ. Suppression of hepatocyte proliferation by hepatocyte nuclear factor 4alpha in adult mice. J Biol Chem. 2012:287:7345-56.

38. Ning BF, Ding J, Yin C, Zhong W, Wu K, Zeng X, et al. Hepatocyte nuclear factor 4 alpha suppresses the development of hepatocellular carcinoma. Cancer res. 2010;70:7640-51. 
39. Spath GF, Weiss MC. Hepatocyte nuclear factor 4 provokes expression of epithelial marker genes, acting as a morphogen in dedifferentiated hepatoma cells. J Cell Biol. 1998;140:935-46.

40. Langmead B, Trapnell C, Pop M, Salzberg SL. Ultrafast and memory-efficient alignment of short DNA sequences to the human genome. Genome Biol. 2009;10:R25.

41. Li D, Zhang B, Xing X, Wang T. Combining MeDIP-seq and MRE-seq to investigate genome-wide CpG methylation. Methods. 2015;72:29-40.

42. Zhang Y, Liu T, Meyer CA, Eeckhoute J, Johnson DS, Bernstein BE, et al. Model-based analysis of ChIP-Seq (MACS). Genome Biol. 2008;9:R137.

43. Grant CE, Bailey TL, Noble WS. FIMO: scanning for occurrences of a given motif. Bioinformatics. 2011;27:1017-8.

44. Heinz S, Benner C, Spann N, Bertolino E, Lin YC, Laslo P, et al. Simple combinations of lineage-determining transcription factors prime cis-regulatory elements required for macrophage and B cell identities. Mol Cell. 2010;38:576-89.

45. Zhang B, Xing X, Li J, Lowdon RF, Zhou Y, Lin N, et al. Comparative DNA methylome analysis of endometrial carcinoma reveals complex and distinct deregulation of cancer promoters and enhancers. BMC Genomics. 2014;15:868.

Submit your next manuscript to BioMed Central and we will help you at every step:

- We accept pre-submission inquiries

- Our selector tool helps you to find the most relevant journal

- We provide round the clock customer support

- Convenient online submission

- Thorough peer review

- Inclusion in PubMed and all major indexing services

- Maximum visibility for your research

Submit your manuscript at www.biomedcentral.com/submit
Biomed Central 